Mini-review

\title{
Developmental Coordination Disorder (DCD): An overview of the condition and research evidence
}

\author{
Priscila Caçola $^{1}\left(\mathbb{0}\right.$, Guilherme Lage ${ }^{2}(1)$ \\ ${ }^{1}$ University of Texas at Arlington, Department of Kinesiology, Arlington, TX, USA; ${ }^{2}$ Universidade Federal \\ de Minas Gerais, UFMG, Department of Physical Education, Belo Horizonte, MG, Brazil
}

\begin{abstract}
Developmental Coordination Disorder (DCD) is a neurodevelopmental condition marked by impairments in the development of motor coordination. The uncoordinated movements of children with DCD lead to performance difficulties in daily life activities and academic settings. Despite the high prevalence of this condition (2-7\%) and severe consequences associated with it, DCD is not well recognized in clinical and educational practices, particularly in Brazil. This review provides an overview of DCD and the research evidence - we present definitions and characteristics associated with the condition, the diagnostic criteria, associated difficulties, frequent co-morbidities and a summary of the possible causes. Finally, we review management strategies and intervention approaches for DCD. We also discuss some of the common challenges of the field - while DCD has been largely studied in the last decade, there are still many gaps between research and practice that need to be filled. Awareness and dissemination of relevant, scientific information is necessary. In conclusion, DCD is a significant condition with a clear diagnostic criteria, and requires intervention to improve motor and functional skills, which can improve the associated difficulties as well as the physical and mental health consequences of the condition.
\end{abstract}

Keywords: developmental coordination disorder; motor development; children; motor skills; neurodevelopmental disorder

\section{Overview}

Developmental Coordination Disorder (DCD) is a neurodevelopmental condition involving problems in motor coordination that affect activities of daily living and academic achievement ${ }^{1}$. The movements of children with DCD are often described as "clumsy" and "uncoordinated," and frequently lead to performance difficulties that most typically developing (TD) children can perform easily ${ }^{2}$. Currently, DCD is a vibrant and dynamic area of study, and much has been discovered in the last few decades about the mechanisms, interventions, and consequences of this condition. However, little has been disseminated in clinical and educational practices, especially in Brazil. Our goal with this review is to provide an overview of the condition and the research evidence, as well as to point out some of the challenges of the field. With this, we expect to bridge some of the gaps between research and practice and to promote a clear picture of DCD for families, clinicians, medical doctors, educators, and rehabilitation professionals.

DCD may be defined in different ways. In general, it is understood as poor motor proficiency that significantly interferes with daily living activities (it is important to note that the low motor proficiency here needs to be low to the point of interfering with performance in daily living activities). DCD can be explained as difficulties or impairments in the performance of "age-appropriate" activities, and has also been described as problems in motor coordination despite appropriate levels of intelligence. Most people refer to DCD as a general "clumsiness", or children that appear to be "clumsy" or "awkward" in how they move, without any apparent reason. In this paper, we will use the definition created by the CanChild Centre for Childhood Disability Research ${ }^{3}$ (a research center housed by McMaster University, in Canada that studies and provides trusted information on DCD): "DCD is a motor skill disorder that occurs when a delay in the development of motor skills, or difficulty coordinating movements, results in a child being unable to perform common, everyday tasks. By definition, children with DCD do not have an identifiable medical or neurological condition that explains their coordination problems". We selected this definition because it seems to encompass several important aspects of DCD - their broad motor skill difficulties, the disruption of everyday activities, and the lack of another clear condition that explains these difficulties.

\section{Diagnosis}

ADCD diagnosis is made with the basis on the Diagnostic and Statistical Manual of Mental Disorders - 5th edition (DSM-5) ${ }^{1}$. In the manual, DCD is categorized under the umbrella of "neurodevelopmental conditions". The diagnostic process involves the assessment of four criteria: A) Learning and execution of coordinated motor skills is below expected level for age, given opportunities for skill learning; B) Motor skill difficulties significantly interfere with activities of daily living and impact academic/school productivity, prevocational and vocational activities, leisure and play; C) Onset is in the early developmental period; and D) Motor skill difficulties are not 
better explained by intellectual delay, visual impairment or other neurological conditions that affect movement. When contemplating a potential diagnosis of DCD, it is essential to take into consideration these four criteria. Another important organization, the International Classification of Diseases, $10^{\text {th }}$ ed. (ICD-10) ${ }^{4}$, also follows the DSM-5 definition and diagnostic criteria, categorizing DCD as a Specific Developmental Disorder of Motor Function (Code F82), and adding the terms "Clumsy Child Syndrome" and "Developmental Dyspraxia" as applicable to the condition.

Technically, only a medical doctor can diagnose DCD (in general, pediatrician, developmental pediatrician, pediatric neurologist, or neurologist) - however; the identification of motor difficulties and the evaluation of each criterion will, most likely, be performed by a team of professionals, which can include (but are not limited to) teachers, psychologists, neuropsychologists, educational diagnosticians, occupational therapists, physical therapists, etc. It is important to highlight this fact, since health and education professionals will potentially be more involved in the diagnostic pathway of DCD than a medical doctor. A variety and combination of methods should be used to identify DCD - medical history, interview, questionnaires, clinical examination, and motor tests. Assessment tools that measure other domains of development should also be considered, such as those measuring cognitive ability, activities of daily living, participation, emotional function, etc.

DCD tends to be a secondary diagnosis, and the recommendation is that a diagnosis is made only around or after age 6 . However, delays and difficulties are typically observed early in life (Criterion C). While we certainly encourage families to pursue a diagnosis, we would like to state that it is possible (and also recommended) to start motor stimulation and intervention early -before a diagnosis is given. Parents should seek appropriate interventions and discuss options with the child's doctor as soon as delays in motor development are observed. In addition, it is important to note that while parents and family members may observe signs of DCD early in life, children may or may not present delays in typical developmental milestones (crawling, walking, talking). Developmental milestones are often considered a measure of typical development, but, in the case of DCD, not having delays in milestones is still possible. The delays seen in Criterion C are commonly related to acquisition of motor skills - such as using silverware, dressing, playing with a toy, scribbling with a crayon, etc.

From an assessment standpoint, DCD is generally evaluated with the basis on an individual's performance in the following broad categories: Manual Dexterity, Balance, and Aiming \& Catching (ball skills). These are the components of the Movement Assessment Battery for Children, $2^{\text {nd }}$ edition (MABC- 2$)^{6}$. The MABC-2 is an assessment whose goal is to identify, describe, and guide treatment of motor impairment in children aged between 3- and 16 years. Throughout the years, the MABC has become the gold standard assessment for DCD, indicating that children scoring below the $5^{\text {th }}$ (red zone) or even the $16^{\text {th }}$ (amber zone) percentiles have motor difficulties significant enough that they could be considered for a potential diagnosis of DCD. It is also possible that children demonstrate difficulties in one, two, or all of the three areas of the MABC-2. No child with DCD is the same and DCD is a highly heterogeneous condition. In addition, other assessments may be used to identify motor impairment, and the second most used in the case of DCD (worldwide) is the Bruininks-Oserestsky Test of Motor Proficiency, $2^{\text {nd }}$ edition (BOT-2) ${ }^{7}$. Obviously, the choice of assessment will depend on accessibility, familiarity, and cultural appropriation of the items. However, the recommendation is that at least one standardized assessment of motor development is used during the evaluation process.

Despite the visible differences in the motor behavior of children with DCD (when compared to typically developing children), often times their difficulties are dismissed as behavioral problems, especially if they are accompanied by a child's frustration and anger. One of the characteristics of DCD is that the motor impairment exists despite the absence of intellectual deficits - which is not to say that children with DCD do not have difficulties associated with cognitive mechanisms, such as executive functioning and working memory ${ }^{8}$. This leads us to the notion that children with DCD are highly aware of their motor difficulties and the fact that they are different from their peers - obviously, they may not understand why they have such difficulties, and why their best attempts to perform a task are often clumsy. Because of that, it is not unreasonable to respond with anger and frustration, which can many times be labeled as behavioral issues.

In fact, children with DCD seem to experience a broad number of difficulties. That is not surprising, given the fact that the motor system underlies everything (every task) we do - walking, reaching for objects, getting dressed, writing, typing, driving - all daily living skills that require a significant amount of motor proficiency. The motor system underlies everything we do, and also serve as a platform of how we interact with the world. That is the very reason why children with DCD have difficulties that go beyond playing sports and participating in physical education classes. They tend to move awkwardly and have poor postural control, being more prone to trip, bump into things, fall, and also appear to move slower and have "delayed" actions and responses. As previously mentioned, they have difficulties with daily living skills and academic tasks. They also put more effort in order to accomplish skills, and have difficulties creating solutions for movements and adjusting/ adapting to different demands of the environment. Consequently, they often withdraw from participating in physical activities, especially those that require a minimum level of proficiency in motor skills.

\section{Other associated difficulties \& co-morbidities}

As previously mentioned, the core deficit of DCD is on the motor system and the difficulties associated with the condition are visible when the individual tries to perform coordinated skills, but there are many deficits that are also associated with the condition. For example, children with DCD have been shown to have difficulties in executive functioning (working memory, inhibition, planning, and fluency) ${ }^{8}$, mathematical performance ${ }^{9}$, 
planning for end-state comfort ${ }^{10}$, oculomotor processes ${ }^{11}$, motor imagery ${ }^{12}$, and others. While the diagnostic criteria for DCD specify that movement difficulties must be below the expected level for the individual's age, and cannot be better explained by an intellectual disability, children with DCD tend to perform worse than their peers on several IQ indicators ${ }^{13}$. More than often, these difficulties are associated with other symptoms, such as anxiety and depression. DCD has also been associated with mental health issues later in life ${ }^{14}$. In addition, quality of life of these children has been shown to be severely impaired, and even lower than that of children living with chronic health conditions (such as cancer) ${ }^{15}$.

Another associated concern for DCD is the link between motor skill competence and physical activity levels. As motor coordination is essential for engagement in physical activity across the lifespan ${ }^{16}$, motor impairments during childhood and through emerging adulthood may subsequently impact engagement in physical activities, leading to a variety of secondary consequences associated with physical health, such as obesity or poor cardiovascular function ${ }^{17}$. Children with DCD are less likely to be physically active and have excess weight gain - and this finding has also been confirmed in a longitudinal study, where DCD was associated with higher body mass and waist circumference over time ${ }^{18}$.

Building on the association between DCD and health consequences, the Environmental Stress Hypothesis (ESH) was developed in order to examine the effects of primary and secondary stressors associated with $\mathrm{DCD}^{19}$. One of the more novel aspects of the ESH concerns the inclusion of physical inactivity (i.e., lower levels of participation in physical activity including organized sport and active free play, or more time spent in sedentary behaviors such as increased use of screen time) and obesity as potential mediating factors connecting DCD to internalizing problems in children ${ }^{20}$. The confirmation of this hypothesis and current findings suggest that interventions for DCD should also focus on physical and psychosocial well-being, such as participation in physical activities and the establishment of global self-worth.

Even though DCD is associated with several other difficulties, studies have reported that a significant number of children diagnosed with DCD have also been diagnosed with other developmental disorders, such as learning difficulties ${ }^{9}$, dyslexia $^{21}$, and attention deficit hyperactivity disorder (ADHD) ${ }^{22}$. In addition, the co-occurrence with Autism Spectrum Disorders (ASD) has been recently explored and reported to be high ${ }^{23}$. In fact, comorbidity in DCD is considered to be the "rule" rather than the "exception" 14 , and it is essential for researchers and practitioners to be aware of this fact. In a recent study involving a sample of 96 families of children with DCD, parents reported on average 3 co-morbidities ( 2 in addition to DCD) for their children, and not even one reported a sole diagnosis of DCD for their child ${ }^{15}$.

\section{Causes and current perspective}

Research and discussion in DCD has increased significantly in clinical and field settings, and there is currently a lot of interest in the field. One of the reasons for that is the fact that DCD significantly impacts learning - especially learning in typical classroom and educational settings. As previously mentioned, many school-related activities require a high level of motor function. For example, $42 \%$ of the time during the school day is spent on paper-and-pencil tasks, with the time increasing throughout the grades ${ }^{24}$. Mastering these activities can be frustrating, and can lead to the false notion that children with DCD are not compliant as other children. That is why DCD is still considered a "puzzle" by many - and often times, as previously mentioned, their issues are identified as behavioral problems, rather than a consequence of their motor difficulties.

Part of the DCD "puzzle" comes from the fact that DCD is a very heterogeneous condition, with distinctions in the type and level of motor skill impairment of each individual. This variety in patterns supports the notion that there are underlying neurodevelopmental mechanisms associated with this condition ${ }^{25}$. Recently, several studies have revealed fundamental differences in brain activation patterns in children with DCD when compared to typically developing controls (for a detailed review, see Brown-Lum \& Zwicker ${ }^{26}$ ). In summary, it appears that children with DCD show increased activation in certain areas of the brain (for example, fronto-central regions) while performing some tasks, or under activation in certain areas (for example, right Dorsolateral Prefrontal Cortex) when handling different tasks. A recent study using functional Near-Infrared Spectroscopy (fNIRS) found that patterns of cortical activation are task-specific, with differences in the right Pre-Motor Cortex (Pre-MC) and Supplementary Motor Area (SMA) for the curve tracing task of the MABC-2, and the right Dorsolateral Prefrontal Cortex (DLPFC) and the right Pre-MC for the paragraph writing task ${ }^{27}$. According to Brown-Lum and Zwicker ${ }^{26}$, a combination of these findings supports the hypothesis that DCD is the result of atypical brain development, and establish the notion that children with DCD are neurobiologically different than TD peers.

From another perspective, Wilson, Smits-Engelsman, Caeyenberghs, Steenbergen, Sugden, Clark, Mumford, Blank ${ }^{28}$ explain that studies exploring brain activation in this population have very small sample sizes with limited behavioral or missing data, do not correct for multiple comparisons or global brain metrics, and do not account for the confounding effects of demographic variables, among other problems. These authors argue that because the studies present methodological flaws, it is still not possible to make a formal conclusion about the neural basis of DCD. However, we believe that work in this area will continue to develop and improve, and conclusions will support the current evidence - that there are fundamental differences in how the brains of individuals with DCD function, and that is the reason why they display behavioral differences in how they plan and execute motor skills.

Another strong body of literature has linked the potential cause of DCD to deficits in planning motor actions, and, confirming that hypothesis, several studies have documented deficits in motor imagery in children with $\mathrm{DCD}^{10}$. Motor imagery (MI) refers to the imagination of a motor task without actual movement execution ${ }^{29}$, and is believed to represent one's ability to accurately utilize forward internal models of motor control. Internal models 
provide stability to the motor system by predicting the outcome of movements before sensorimotor feedback is available. Without that ability, movements are clumsy and disorganized, which explains most of the problems seen in DCD.

However, another line of research proposes that DCD is a result of a deficit in the relationship between perception and action ${ }^{30}$. These authors explain that "learning how to control movements and coordinate body segments is dependent primarily on one's sensory experience and whether an individual correctly detects (or is sufficiently sensitive to) information". This perspective challenges the computational, cognitive approach that focuses on the planning and execution of motor skills as a closed and cyclical loop. The advantage lies in the fact that this approach may provide a more complete picture of the problems in DCD, because it takes into account the relationship between task demands and the environmental context. However, more studies are needed for the causes of DCD to be fully understood - nonetheless, we believe that the current perspectives enhance our knowledge of the condition, and give important clues about its management and intervention.

\section{Management \& Intervention}

Obviously, DCD is a condition that needs management and intervention. In general, interventions for DCD are not supposed to work as "treatment", as the condition has no cure. But interventions can provide skills, strategies, and accommodations to make it easier for children with DCD to execute motor tasks required in daily living activities and school environments. From a broad perspective, Missiuna and colleagues ${ }^{31}$ suggest that some interventions should target the population level, creating environments that facilitate the learning of motor skills, function and participation for all children. This perspective states that it is important to take the focus out of the child, and instead place the focus on what can be adapted in the surroundings so the child can perform the task(s) successfully.

However, when practitioners want to improve a child's motor skills, there are several types of protocols available. A review of the literature suggests that task-based approaches, which focus on finding solutions for tasks at hand, yield stronger effects on improving functional outcomes when compared to process-based approaches, which practice a variety of abilities and skills. The American Academy of Pediatrics also released a policy statement in 2012 recommending that the use of therapies that are process-based (such as sensory-based therapies) may be acceptable as part of a comprehensive treatment plan, but there should be a priority for treatments based on problems that affect a child's ability to perform daily functions ${ }^{32}$. However, we wish to note that there has not been an update on this policy since 2012 .

A new systematic review has extended support for the effectiveness of activity-oriented (task-oriented) intervention approaches, as well as body function-oriented approaches when combined with activity-oriented and active video games interventions ${ }^{33}$. They also found evidence of training effectiveness when programs were conducted in a small group format and for programs aiming at improving physical fitness ${ }^{33}$. Overall, these findings established that approaches designed to improve targeted body functions considered to underlie the reported functional motor problem, when combined with other approaches, can be effective. It shows that relatively new types of approaches, such as those involving active video games may be a feasible option to increase motivation and avoid boredom. The authors concluded by describing the importance of training everyday activities by targeting the weakest link in the activity chain, as well as the participation in programs that support learning of complex motor skills in the areas that are of the child's interest.

\section{Challenges \& Conclusions}

There are plenty of challenges when it comes to the understanding, diagnosis, and intervention for DCD. First and foremost, awareness of the condition is still far from ideal. Despite its high prevalence (up to $7 \%$ of the school-age population), DCD is still not as recognized as other conditions, such as Autism and ADHD, which are disorders with a much lower prevalence than DCD. It is essential to increase and facilitate awareness of this disorder, and to share and propagate scientific and relevant information to educators and health professionals, including medical doctors - unfortunately, a study that promoted an online survey for 1,297 parents, teachers and physicians found that only $41 \%$ of pediatricians and $23 \%$ of general practitioners had any knowledge of the condition (in Canada, US, and United Kingdom). Furthermore, only $23 \%$ of the pediatricians and $9 \%$ of the general practitioners surveyed had ever diagnosed DCD. The vast majority of physicians reported the need for more education about the condition ${ }^{34}$.

Another challenge relates to the understanding, prevention, and management of the long-term outlook and consequences of the condition. DCD is a chronic condition, but little is known about its effects on adult life, and what types of interventions are effective for individuals that may suffer the consequences of DCD (even without a clear diagnosis). We can also add many more challenges to this list - accessibility to diagnostic and intervention services, follow-up of these services, need for parent education, research (and research funding) aimed at the understanding of the causes, mechanisms, interventions, and consequences of the condition, etc. However, on a positive and optimistic note, studies in the field and awareness of the condition have come a long way in the last decade. In 2011, new guidelines were announced during the 9th International Conference on DCD, and later posted on the European Academy of Childhood Disability (EACD) website ${ }^{35}$. These guidelines have just been updated and are now published in the Developmental Medicine and Child Neurology Journal ${ }^{36}$.

In conclusion, DCD is a highly prevalent and co-morbid condition, and children with DCD represent a significant portion of the school-aged population. We determined that motor difficulties in DCD are associated with several and severe consequences in performance of activities of daily living, 
academic achievement, as well as problems in physical and mental health. We also provided an overview of the causes and recent research in the field. Here, it is important to understand that there are no single causes, profile, and known cure for the condition $^{2}$. Finally, it is essential to increase awareness of the condition, and promote interventions that focus on improving functional skills and a child's environment.

\section{References}

1. American Psychiatric Association, editors. Diagnostic and Statistical Manual of Mental Disorders. 5th ed. Washington, American Psychiatric Association, 2013.

2. Caçola P. Movement difficulties affect children's learning: An overview of Developmental Coordination Disorder (DCD). Learn Disabil Multidiscip J. 2014;20(2):98-106. doi: 10.18666/ LDMJ-2014-V20-I2-5279.

3. CanChild. Developmental Coordination Disorder. Available from: https://www.canchild.ca/en/diagnoses/developmental-coordination-disorder [Acessed 10th November 2018].

4. Center for Disease Control and prevention. International Classification of Diseases, 10th ed. (ICD-10). Available from: https://www.cdc.gov/ nchs/icd/icd10cm.htm [Acessed 10th November 2018].

5. Blank R, Smits-Engelsman B, Polatajko H, Wilson P. European Academy for Childhood Disability (EACD): Recommendations on the definition, diagnosis and intervention of developmental coordination disorder (long version). Dev Med Child Neurol. 2012;54(1):54-93. doi: 10.1111/j.1469-8749.2011.04171.x.

6. Henderson SE, Sugden DA, Barnett AL, editors. Movement assessment battery for children - second edition [MABC-2], 2nd ed. London, The Psychological Corporation, 2007.

7. Bruininks R, Bruininks B, editors. Bruininks-Oseretsky test of motor proficiency - second edition: Manual. Circle Pines, AGS Publishing, 2005.

8. Leonard HC, Hill EL. Executive Difficulties in Developmental Coordination Disorder: Methodological Issues and Future Directions. Curr Dev Disord Rep. 2015;2(2):141-49. doi: 10.1007/ s40474-015-0044-8.

9. Gomez A, Piazza M, Jobert A, Dehaene-Lambertz G, Dehaene S, Huron C. Mathematical difficulties in Developmental Coordination Disorder: Symbolic and nonsymbolic number processing. Res Dev Disabil. 2015;43-44:167-78. doi: 10.1016/j.ridd.2015.06.011.

10. Adams IL, Lust JM, Wilson PH, Steenbergen B. Development of motor imagery and anticipatory action planning in children with Developmental Coordination Disorder- A longitudinal approach. Hum Mov Sci. 2017;55:296-306. doi: 10.1016/j.humov.2017.08.021.

11. Sumner E, Hutton SB, Kuhn G, Hill EL. Oculomotor atypicalities in Developmental Coordination Disorder. Dev Sci. 2018;21:e12501. doi: 10.1111/desc.12501.

12. Fuchs CT, Caçola P. Differences in accuracy and vividness of motor imagery in children with and without Developmental Coordination Disorder. Hum Mov Sci. 2018;60:234-41. doi: 10.1016/j.humov.2018.06.015.

13. Sumner E, Pratt ML, Hill EL. Examining the cognitive profile of children with Developmental Coordination Disorder. Res Dev Disabil. 2016;56:10-7. doi: 10.1016/j.ridd.2016.05.012.
14. Lingam R, Jongmans MJ, Ellis M, Hunt LP, Golding J, Emond A. Mental health difficulties in children with Developmental Coordination Disorder. Pediatrics. 2012;129(4):e882-e891. doi: 10.1542/peds.2011-1556.

15. Caçola P, Killian M. Health-related quality of life in children with Developmental Coordination Disorder: Association between the PedsQL and KIDSCREEN instruments and comparison with their normative samples. Res Dev Disabil. 2018;75:32-9. doi: 10.1016/j.ridd.2018.02.009.

16. Stodden DF, Goodway JD, Langendorfer SJ, Roberton MA, Rudisill ME, Garcia C. Garcia LE. A developmental perspective on the role of motor skill competence in physical activity: An emergent relationship. Quest, 2008;60(2):290-306. doi: 10.1080/00336297.2008.10483582.

17. Cairney J, Veldhuizen S. Is developmental coordination disorder a fundamental cause of inactivity and poor health-related fitness in children? Dev Med Child Neurol. 2013;55(4):55-8. doi: 10.1111/dmcn. 12308 .

18. Joshi D, Missiuna C, Hanna S, Hay J, Faught BE, Cairney J. Relationship between BMI, waist circumference, physical activity and probable developmental coordination disorder over time. Hum Mov Sci, 2015;40:307-317. doi: 10.1016/j.humov.2014.12.011.

19. Cairney J, Rigoli D, Piek J. Developmental Coordination Disorder and internalizing problems in children: The environmental stress hypothesis elaborated. Dev Rev. 2013;33(3):224-238. doi: 10.1016/j.dr.2013.07.002.

20. Li Y, Kwan MYW, Clark HJ, Hay J, Faught BE, Cairney J. A test of the Environmental Stress Hypothesis in children with and without Developmental Coordination Disorder. Psychol Sport Exerc. 2018;37:244-250. doi: 10.1016/j.psychsport.2017.11.001.

21. Biotteau M, Péran P, Vayssière N, Tallet J, Albaret J-M, Chaix Y. Neural changes associated to procedural learning and automatization process in Developmental Coordination Disorder and/or Developmental Dyslexia. Eur J Paediatr Neurol. 2017;21(2):286-299. doi: 10.1016/j.ejpn.2016.07.025.

22. Goulardins JB, Rigoli D, Licari M, Piek JP, Hasue RH, Oosterlaan J, Oliveira JA. Attention Deficit Hyperactivity Disorder and Developmental Coordination Disorder: Two separate disorders or do they share a common etiology. Behav Brain Res. 2015;292:484-492. doi: 10.1016/j.bbr.2015.07.009.

23. Caçola P, Miller HP, Ossom-Williamson P. Behavioral comparisons in Autism Spectrum Disorder and Developmental Coordination Disorder: A systematic literature review. Res Autism Spectr Disord. 2017;38:6-18. doi: 10.1016/j.rasd.2017.03.004.

24. Marr D, Cermak S, Cohn ES, Henderson A. Fine motor activities in Head Start and kindergarten classrooms. Am J Occup Ther. 2003;57:550-557. doi:10.5014/ajot.57.5.550.

25. Wilson PH, Ruddock S, Smits-Engelsman B, Polatajko H, Blank R. Understanding performance deficits in Developmental Coordination Disorder: a meta-analysis of recent research. Dev Med Child Neurol. 2013;55(3):217-228. doi: 10.1111/j.1469-8749.2012.04436.x.

26. Brown-Lum M, Zwicker JG. Brain imaging increases our understanding of Developmental Coordination Disorder: a review of literature and future directions. Cur Dev Disord Rep. 2015;2(2):131-140. doi: 10.1007/s40474-0150046-6.

27. Caçola P, Getchell N, Srinivasan D, Alexandrakis G, Liu H. Cortical activity in fine-motor tasks in children with Developmental 
Coordination Disorder: A preliminary fNIRS study. 2018;65:83-90. doi: 10.1016/j.ijdevneu.2017.11.001.

28. Wilson PH, Smits-Engelsman B, Caeyenberghs K, Steenbergen B, Sugden DA, Clark JE, Mumford N, Blank R. Cognitive and neuroimaging findings in Developmental Coordination Disorder: New insights from a systematic review of recent research. Dev Med Child Neurol. 2017;59(11):1117-29, doi: 10.1111/dmcn.13530.

29. Decety J, Grèzes J. The power of simulation: Imagining one's own and other's behavior. Brain Res. 2006;1079(1):4-14. doi: org/10.1016/j.brainres.2005.12.115.

30. Wade MG, Kazeck M. Developmental coordination disorder and its cause: The road less travelled. Hum Mov Sci. 2018;57:489-500. doi: 10.1016/j.humov.2016.08.004.

31. Missiuna CA, Pollock NA, Levac DE, Campbel WN, Whalen, DS, Bennett SM, et al. Partnering for Change: An innovative schoolbased occupational therapy service delivery model for children with developmental coordination disorder. Can J Occup Ther. 2012;79(1):41-50. doi:10.2182/cjot.2012.79.1.6.

32. American Academy of Pediatrics. Sensory Integration therapies for children with developmental and behavioral disorders. Section on Complementary and Integrative Medicine, Council on Children with Disabilities. Pediatrics. 2012;129(6):1186-89. doi: 10.1542/peds.2012-0876.

33. Smits-Engelsman B, Vinçon S, Blank R, Quadrado VH, Polatajko HJ, Wilson PH. Evaluating the evidence for motor-based interventions in developmental coordination disorder: A systematic review and meta-analysis. Res Dev Disabil. 2018;74:72-102. doi: 10.1016/j.ridd.2018.01.002.

34. Wilson BN, Neil K, Kamps PH, Babcock S. Awareness and knowledge of developmental co-ordination disorder among physicians, teachers and parents. Child Care Health Dev 2013;39(2):296-300. doi: 10.1111/j.1365-2214.2012.01403.x.

35. Blank R, Smits-Engelsman B, Polatajko H, Wilson P. European Academy for Childhood Disability (EACD): Recommendations on the definition, diagnosis and intervention of developmental coordination disorder (long version). Dev Med Child Neurol. 2012;54(1):54-93. doi: 10.1111/j.1469-8749.2011.04171.x.

36. Blank R, Barnett A, Carney J. et al. European International clinical practice recommendations on the definition, diagnosis, assessment, intervention, and psychosocial aspects of developmental coordination disorder. Dev Med Child Neurol. 2019;6(13): 242-285. doi: 10.1111/dmen. 14132

\section{Corresponding author}

Priscila Caçola, Ph.D.

Associate Professor, Associate Chair

Department of Kinesiology

University of Texas at Arlington

Email: cacola@uta.edu

Manuscript received on November 19, 2018

Manuscript accepted on December 10, 2018

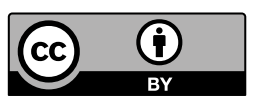

Motriz. The Journal of Physical Education. UNESP. Rio Claro, SP, Brazil - eISSN: 1980-6574 - under a license Creative Commons - Version 4.0 